\title{
Simulation of the Ondes Martenot ribbon-controlled oscillator using energy-balanced modelling of nonlinear time-varying electronic components
}

\author{
Judy NAJNUDEL, \\ judy.najnudel@ircam.fr \\ AES student member \\ Conservation Recherche team, CNRS-Musée de la Musique and S3AM team, STMS laboratory, IRCAM-CNRS-SU \\ Thomas HELIE, \\ thomas.helie@ircam.fr \\ AND David ROZE \\ david.roze@ircam.fr \\ CNRS, S3AM team, STMS laboratory, IRCAM-CNRS-SU
}

\begin{abstract}
The Onde Martenot is a classic electronic musical instrument. This paper focuses on the power-balanced simulation of its ribbon-controlled oscillator, composed of linear, nonlinear as well as time-varying components. To this end, the proposed approach consists in formulating the circuit as a Port-Hamiltonian System, for which power-balanced numerical methods are available. A specificity of the Martenot oscillator is to involve parallel capacitors, one of them having a capacitance which non-linearly depends on the time-varying ribbon position state. In the case of linear time-invariant (LTI) capacitors in parallel, an equivalent component can be deduced using the classic impedance approach. Such a reformulation into a single equivalent component is required to derive a state-space Port-Hamiltonian representation of a circuit. One technical result of this paper is to propose a method to determine such an equivalent component in the non LTI case. This method is applied to the present Martenot oscillator. Then, power-balanced numerical experiments are presented for several configurations: fixed ribbon position, realistic and over-speed movements. These results are examined and interpreted from both the electronic and mechanical points of view.
\end{abstract}

\section{Introduction}

As the audio industry is moving towards the digital era, the question of the preservation of analog machines and instruments is paramount. This question is especially relevant for the Onde Martenot, one of the first electronic musical instruments [1] invented in 1928, for it is no longer produced and some of its components are now obsolete. A satisfying solution consists in modelling its circuit in order to build a virtual instrument, so that the community of composers, musicians and musicologists may at least have access to facsimile. To model electronic circuits for audio applications, the state-space form known as Port-Hamiltonian Systems (PHS) has proven to be a powerful approach as it guarantees the power balance of the considered system, therefore preserves the passivity of simulations [2] even when its components are not linear. It is multi-physical (a system can be electrical, mechanical, thermal or a mix as well) and modular (a system made of several connected PHS is still a PHS). Yet for some circuit configurations, a direct statespace form cannot be derived - the circuit is said not to be realizable - and an equivalent circuit must be computed in order to perform simulations. This is the case with parallel capacitors which must be replaced by a single equivalent capacitor. However, when the components involved in the circuit are not LTI, as some are in the controllable oscillator of the Onde Martenot, the classic impedance approach is no longer suitable and equivalent components must be computed through a specifically designed method.

This paper is structured as follows: the Martenot controllable oscillator circuit is presented in section 2 , with a par- 
ticular attention drawn to the realization problem it poses. In section 3, the PHS formalism is briefly described. In section 4 , a method to compute equivalent components in this formalism is developed. A modelling of the complete oscillator is then derived, and several configurations are simulated in section 5. Finally the simulation results are discussed in section 6 .

\section{Ondes Martenot ribbon-controlled oscillator and problem statement}

\subsection{Circuit overview}

The Onde Martenot, invented by Maurice Martenot in 1928, is one of the first electronic musical instruments and is based on heterodyne processing. Heterodyning is a technique used to shift high frequency signals into the audio domain. In the Onde Martenot specifically, each one of two oscillators generate a high frequency quasi-sinusoidal voltage (around $80 \mathrm{kHz}$ ); one is fixed, and the player controls the second frequency using a sliding ribbon. The sum of these two voltages is an amplitude-modulated signal. Its envelope is detected using a triode vacuum tube, producing an audible sound, for which the frequency is the difference between the two oscillators frequencies. The triode vacuum tube in the detector is a nonlinear component which adds harmonics to the signal. This enriched signal is then routed towards special kinds of loudspeakers (called diffuseurs) selected by the musician, adding another layer of coloration to the sound. The oscillators are made of an LC circuit cou-

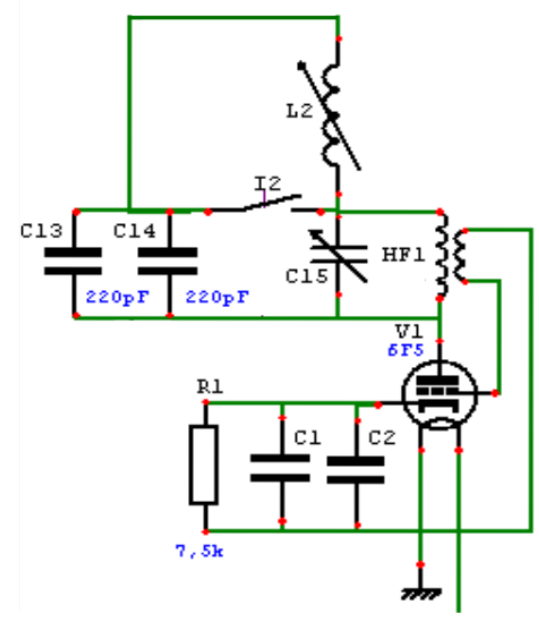

Fig. 1. Schematic of the Onde 169 controllable oscillator (source: Musée de la Musique, Paris).

pled to a triode vacuum tube (for amplification), through a transformer. In the controllable oscillator, one of the capacitors of the LC circuit is variable and controlled by the ribbon. Fig. 1 shows that the total capacitor is in fact made of several capacitors connected in parallel, some of them LTI, but one of them time-varying.

\subsection{Problem statement}

Two capacitors $C_{A}$ and $C_{B}$ connected in parallel are equivalent to a single capacitor $C_{C}$ (fig. 2). In the case of a Linear
Time-invariant system, the notion of impedance allows to determine the equivalent capacitor. Indeed, denoting the capacitors impedances $Z_{A}=\frac{1}{j C_{A} \omega}$ and $Z_{B}=\frac{1}{j C_{B} \omega}$ respectively, Kirchoff's laws $i_{C}=i_{A}+i_{B}$ and $v_{C}=v_{A}=v_{B}$ yield the relation

$$
\frac{1}{Z_{C}}=\frac{1}{Z_{A}}+\frac{1}{Z_{B}}
$$

This relation characterizes entirely the equivalent component $C_{C}$ and gives the value of its capacitance:

$$
j C_{C} \omega=j C_{A} \omega+j C_{B} \omega \Rightarrow C_{C}=C_{A}+C_{B}
$$

However, this classic impedance approach is no longer suitable for non LTI systems: if we were to naively define impedance by the ratio $v / i$ (transfer function), that of nonlinear capacitors would still depend on the charge $q$, itself time-dependent. By definition, time-varying capacitors would also yield a time-dependent transfer function. As the ribbon-controlled capacitance depends on the ribbon position, which itself depends on time, an adapted method to characterize the equivalent capacitor is needed. The PHS formalism allows to represent an energy-storing component by its energy function instead of its impedance. We thus may rely on this notion in a non LTI case, as it is more general.

\section{Port-Hamiltonian Systems: formalism and examples}

This section recalls basics on port-Hamiltonian systems (PHS).

For detailed presentation, readers can refer to [3] and [4].

\subsection{Formalism}

Here we rely on a differential-algebraic form adapted to multi-physical systems [5] [6], which allows to represent a dynamical system as a network of storage components with their state variable $\mathbf{x}$ and total energy of the state $H(\mathbf{x})$, dissipative components with their variable $\mathbf{w}$ and constitutive law $z(\mathbf{w})$, and connection ports as control inputs $\mathbf{u}$ and their associated outputs $\mathbf{y}$ such as $\mathbf{u}^{\top} \mathbf{y}$ is the external power brought to the system. The variables are generally time-dependent and can be vectors. If such a system is realizable [7] [8], the flows and efforts exchanges between the system components are coupled through a skew-symmetric

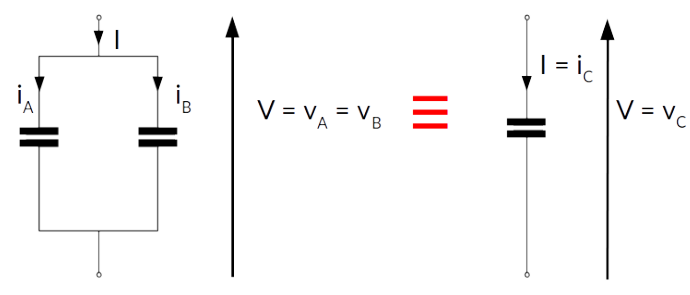

Fig. 2. Equivalence between two parallel capacitors and a single capacitor. 
matrix $S=-S^{\top}$ :

$$
\underbrace{\left(\begin{array}{c}
\frac{d x}{d t} \\
w \\
-y
\end{array}\right)}_{\mathscr{F}(\text { flows })}=S \cdot \underbrace{\left(\begin{array}{c}
\nabla H(x) \\
z(w) \\
u
\end{array}\right)}_{\mathscr{E}(\text { efforts })}
$$

The skew-symmetry of $S$ guarantees that the system remains passive, i.e there is no spontaneous creation of energy. Indeed, from Eq. (3), the scalar product of the efforts and flows yields

$$
\begin{aligned}
\mathscr{E} \top \mathscr{F} & =\mathscr{E}^{\top} S \mathscr{E}=\left(\mathscr{E}^{\top} S \mathscr{E}\right)^{\top}=-\mathscr{E}^{\top} S \mathscr{E} \\
& =-\mathscr{E}^{\top} \mathscr{F}=0,
\end{aligned}
$$

meaning that the following power-balance is satisfied

$$
\underbrace{\frac{d E}{d t}}_{\nabla H(x)^{\top} \frac{d x}{d t}}=\underbrace{P_{e x t}}_{u^{\top} y}-\underbrace{P_{\text {diss }}}_{z(w)^{\top} w \geq 0},
$$

where $E=H(x)$ is the energy, $P_{\text {ext }}$ is the (incoming) external power and $P_{\text {diss }} \geq 0$ the dissipated power. Appendix A.2 and [2] describe a numerical scheme preserving those properties in discrete time.

\subsection{Capacitors constitutive laws}

For linear time-invariant capacitors, the charge $q$ and the voltage $v$ are mapped according to a constitutive law $q=C v$, which depends on a unique characteristic constant (capacitance $C$ in Farad). The electric power $P=i v$ received by such a component makes its stored energy $E$ vary as $\frac{\mathrm{d} E}{\mathrm{~d} t}=P$. With current $i=\frac{\mathrm{d} q}{\mathrm{~d} t}$, voltage $v=\frac{q}{C}$ and assuming a zero energy for a discharged component, a time integration yields $E=H(q)$ with $H(q)=\frac{q^{2}}{2 C}$. This energy is sometimes expressed independently of value $C$ as $E=\frac{q v}{2}$.

For nonlinear capacitors, the last expression in no longer true. But a description based on an energy function $q \mapsto$ $H(q)$ is still applicable. The constitutive law is described by the voltage function $H^{\prime}$ (derivative of $H$ ), namely,

$$
v=H^{\prime}(q) \text {, }
$$

with power balance $v i=H^{\prime}(q) \frac{\mathrm{d} q}{\mathrm{~d} t}=\frac{\mathrm{d} H(q)}{\mathrm{d} t}=\frac{\mathrm{d} E}{\mathrm{~d} t}$.

Remark 1 (Constitutive laws based on q or v). In practice, constitutive laws are usually formulated (and measured) with respect to the voltage (effort) rather than the charge (state). Formally (if possible), such a description corresponds to invert $v=H^{\prime}(q)(=q / C$ for linear capacitors $)$ into $q=F(v)$ ( $=C v$ for linear capacitors) with $F=H^{\prime-1}$ and formulates the energy as $E=H\left(H^{\prime-1}(v)\right)=\frac{C v^{2}}{2}$. Note that differentiating this formula yields a (correct) power balance but more difficult interpretations.

This remark applies to varactors proposed in [9], with model $H^{\prime-1}=F: v \mapsto C v / \sqrt{1+v / v_{2}}$. Another example is tanh type which is of the form $q \mapsto H^{\prime}(q)=v_{1} \tanh \left(\frac{q}{q_{0}}\right)$. Fig. 3 shows the voltage functions and the corresponding energy functions of these different capacitors types (for $q_{0}$ $=7 \mathrm{nC}, v_{1}=80 \mathrm{mV}, C=50 \mathrm{nF}$ and $\left.v_{2}=0.2 \mathrm{~V}\right)$.
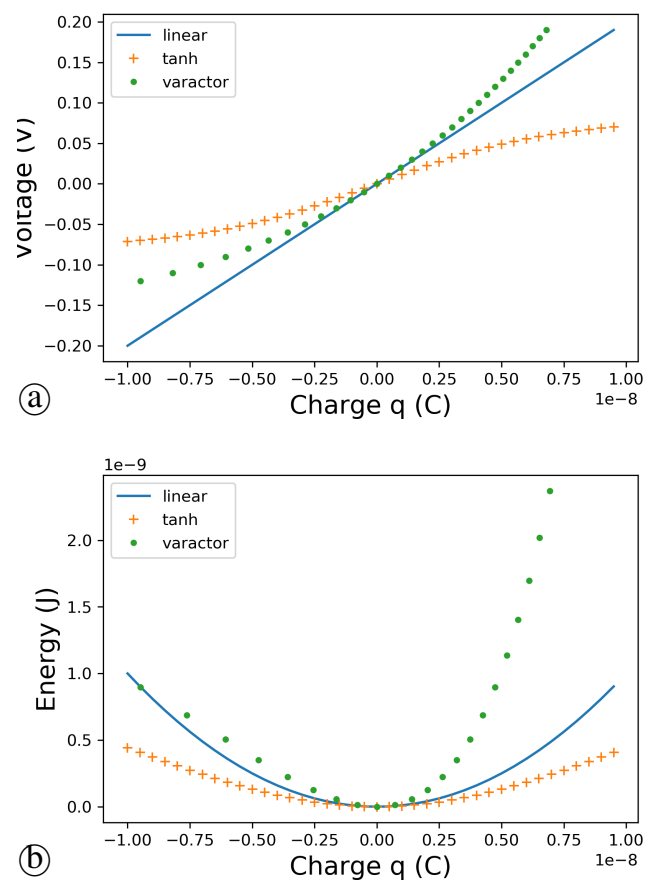

Fig. 3. (a) Voltage functions $H^{\prime}$ (associated with constitutive law Eq. (6)) and (b) energy functions $H$ for different capacitors types.

\section{Equivalent component description of non-LTI parallel capacitors}

\subsection{Method}

\subsection{Problem statement and hypotheses}

Consider two capacitors connected in parallel (fig. 2). These components are flow-controlled $\left(\frac{d x}{d t}=\dot{q}=i\right.$, current). As they are connected in parallel, their dual efforts (voltages) are equal and there is no skew-symmetric matrix $\mathrm{S}$ such that

$$
\left(\begin{array}{c}
i_{A} \\
i_{B} \\
-V
\end{array}\right)=\mathbf{S} \cdot\left(\begin{array}{c}
v_{A} \\
v_{B} \\
I
\end{array}\right),
$$

and formulation (3) cannot be retrieved. Replacing those parallel capacitors by a single equivalent capacitor (with common voltage $v_{C}:=v_{A}=v_{B}$ and total current $i_{C}=$ $i_{A}+i_{B}$ ) allows to restore such a formulation. For linear time-invariant (LTI) laws, the use of transfer functions of impedance-type makes this operation straightforward. For non LTI laws, no characterization can be based on usual transfer functions. In this case, energy-storing components are characterized by their energy function to be used in the PHS formalism.

The purpose of this section is to derive the energy function $H_{C}$ of the equivalent component from the energy functions $H_{k}(k=A, B)$ of isolated components, including for non LTI laws, under the following hypotheses:

(i) the energy function $H_{k}$ is $\mathscr{C}^{1}$ positive-definite $\left(H_{k}(0)=0\right.$ and $H_{k}(x)>0$ for $\left.x \neq 0\right)$ 
(ii) the voltage function $H_{k}^{\prime}$ (derivative of $H_{k}$ ) is strictly increasing and definite $\left(H_{k}^{\prime}(0)=0\right)$

According to Eq. (6), this means that the voltage $v_{k}=$ $H_{k}^{\prime}\left(q_{k}\right)$,

continuously and strictly increases with the charge $q_{k}$, and is zero for a zero charge. In particular, the constitutive law Eq. (6) makes $v_{k}$ and $q_{k}$ in one-to-one relation, allowing therefore its invertibility.

\subsection{Method}

To express the total energy $H_{C}$ as a function of the total charge $q_{C}=q_{A}+q_{B}$ under the constraint that capacitors in parallel share the same voltage $v_{A}=v_{B}=v_{C}$, the method is decomposed into three steps.

Step 1: express the total charge $q_{C}=q_{A}+q_{B}$ as functions of the common voltage $v_{A}=v_{B}=v_{C}$.

From Eq. (6), the charge of isolated components is

$$
q_{k}=H_{k}^{\prime-1}\left(v_{k}\right), \text { for } k=A, B,
$$

so that the total charge depends on the common voltage as

$$
q_{C}=\left[H_{A}^{\prime-1}+H_{B}^{\prime-1}\right]\left(v_{C}\right) .
$$

This function continuously and strictly increases and is zero at zero.

Step 2: express this common voltage $v_{C}$ as a function of the total charge $q_{C}$.

$$
v_{C}=\left[H_{A}^{\prime-1}+H_{B}^{\prime-1}\right]^{-1}\left(q_{C}\right) .
$$

Step 3: express the total energy as a function of $q_{C}$.

The energy values $H_{k}\left(q_{k}\right)$ of elementary components $k=A, B$ can be reformulated as functions of the total charge, using the composed functions $q_{C} \stackrel{(9)}{\longmapsto} v_{C} \stackrel{(7)}{\longmapsto}$ $q_{k}$. Their sum yields the total energy function, that is,

$$
\begin{aligned}
H_{C}\left(q_{C}\right)= & {\left[H_{A} \circ H_{A}^{\prime-1}+H_{B} \circ H_{B}^{\prime-1}\right] } \\
& \circ\left[H_{A}^{\prime-1}+H_{B}^{\prime-1}\right]^{-1}\left(q_{C}\right) .
\end{aligned}
$$

These steps are detailed on examples in appendix A.3.

Remark 2 (Time-varying case). For capacitors that depend on other additional state variables (e.g. the time-varying space variable in Eq. 15, section 4.1.1), steps 1 to 3 are unchanged (these additional variables are considered as parameters in this method).

This is applied to the ribbon controlled-oscillator in section 4.

\subsubsection{Generalizations}

This method can also be extended to $K$ non-LTI capacitors connected in parallel, leading to

$$
H_{\text {tot }}\left(q_{\text {tot }}\right)=\left[\sum_{k=1}^{K} H_{k} \circ H_{k}^{\prime-1}\right] \circ\left[\sum_{k=1}^{K} H_{k}^{\prime-1}\right]^{-1}\left(q_{\text {tot }}\right)
$$

In this case, the charge of each component $k$ is

$$
\begin{aligned}
q_{k} & =H_{k}^{\prime-1}\left(v_{C}\right) \\
& =H_{k}^{\prime-1} \circ\left[\sum_{k=1}^{K} H_{k}^{\prime-1}\right]^{-1}\left(q_{\mathrm{tot}}\right)
\end{aligned}
$$

This method is adapted to other types of storage components that poses similar realization problems. For instance, it is suitable for coils in series, for which the state is the magnetic flux $\phi, i=H^{\prime}(\phi)$ provides the current law and the voltage is $v=\dot{\phi}$. Moreover, when the constitutive laws of the components are not well known, this method can still be used with laws interpolated from measurements. An implementation of the method using piecewise linear functions is available in the PyPHS library [10].

\section{Simulation of the ribbon-controlled oscillator}

\subsection{Ribbon-controlled oscillator modelling}

Constitutive laws of components $R_{1}, L, C_{1}, C_{2}, C_{13}$ and $C_{14}$ are supposed to be linear and described in table 4 , in appendix A.1. The transformer is also supposed to be linear, and of ratio $M$. The remaining components models are described in the next section.

\subsubsection{Equivalent variable capacitor}

The ribbon slides between the faces of a comb-shaped capacitor. As it slides, its conductive part hides and activates complementary parts of the teeth that compose the capacitor, according to the ribbon position (fig. 4). Section 4.2 validates that the LTI capacitors $C_{13}$ and $C_{14}$ and the variable capacitor $C_{15}$ connected in parallel are equivalent to a single variable capacitor. In order to evaluate how the equivalent capacitor behaves with respect to its charge $q$ and the ribbon position $x$, the position is mapped with the heard frequency. The ribbon follows a dummy piano keyboard on which the width

$$
x_{0}=11.10^{-3} \mathrm{~m}
$$

is a semitone [11].
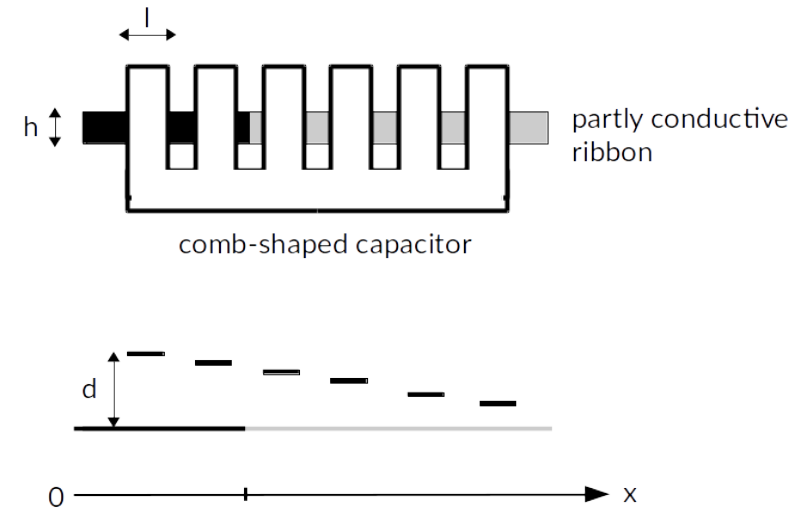

Fig. 4. Variable capacitor of the Onde Martenot with its ribbon control. $\mathrm{h}$ is the ribbon height, $\mathrm{l}$ is the width of a tooth, $\mathrm{x}$ is the ribbon position and $\mathrm{d}$ the distance between the ribbon and the capacitor. 
Denoting $n$ the semitone number where the reference is $A_{1}(110 \mathrm{~Hz}, n=0)$, we roughly have $x=n x_{0}$ (except when the ribbon slides between a $B$ and a $C$ or between an $E$ and a $F$ where the displacement is greater). The Onde Martenot is tuned on equal temperament, therefore the heard frequency from $A_{1}$ is $f_{m}=A_{1} 2^{\frac{x}{12 x_{0}}}$. Denoting $F$ the carrier frequency, the actual oscillator frequency is $f=F-f_{m}$ and the corresponding capacitance of the LC circuit for a static configuration is (ignoring the dissipative effects of the triode vacuum tube for simplicity):

$$
C(x)=\frac{1}{4 \pi^{2}\left(F-A_{1} 2^{\frac{x}{12 x_{0}}}\right)^{2} L} .
$$

Fig. 5 shows $C$ in function of $x$ with $L=2 m H$ and $F=$ $10 \mathrm{kHz}$.

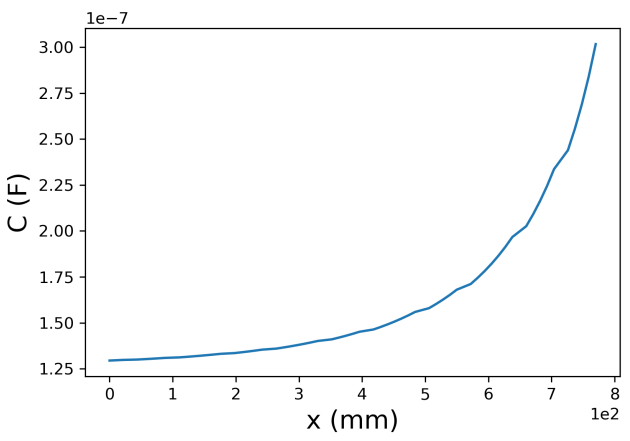

Fig. 5. Capacitance of the variable oscillator in function of the ribbon position from $A_{1}$ to $A_{6}$.

Based on the linear electric behaviour $v=\frac{q}{C(x)}$ observed for any static position $x$, the total energy of the electromechanical component has the form $H(q, x)=H(q=$ $0, x)+\int_{0}^{q} \frac{\xi}{C(x)} \mathrm{d} \xi$. Moreover, the discharged component (then purely mechanical) applies no force $F$ on the ribbon whatever the position $x$. This means that $F=\frac{\partial H}{\partial x}$ is zero at any state $(q=0, x)$, leading to $H(q=0, x)=H(q=$ $0, x=0)=0$ : the discharged component stores no pure mechanical energy. Finally, the internal energy of the electromechanichal component is given by (see also fig. 6)

$$
H_{\text {cap }}(q, x)=\frac{q^{2}}{2 C(x)}
$$

Remark 3 (Energy time-variation). The total energy $E=$ $H(q, x)$ varies as $\frac{\mathrm{d} E}{\mathrm{~d} t}=P_{\mathrm{e}}+P_{\mathrm{m}}$ where

- $P_{\mathrm{e}}=\frac{\partial H}{\partial q}(q, x) \frac{\mathrm{d} q}{\mathrm{~d} t}$ accounts for the incoming electrical power due to current $\frac{\mathrm{d} q}{\mathrm{~d} t}$ and voltage $v=\frac{\partial H}{\partial q}(q, x)=\frac{q}{C(x)}$,

- $P_{\mathrm{m}}=\frac{\partial H}{\partial x}(q, x) \frac{\mathrm{d} x}{\mathrm{~d} t}$ accounts for the incoming mechanical power due to velocity $\frac{\mathrm{d} x}{\mathrm{~d} t}$ and a spring reaction force $F=\frac{\partial H}{\partial x}(q, x)=-\frac{C^{\prime}(x) q^{2}}{2 C(x)^{2}}$, induced by the capacitance variation.

\subsubsection{Triode}

The triode vacuum tube is modelled with an enhanced Norman Koren model [12]. This gives the anode current $i_{p c}$ and grid current $i_{g c}$ in function of the voltages $v_{p c}$ and $v_{g c}$ :

$$
\begin{gathered}
i_{p c}=\left\{\begin{array}{l}
2 E_{1}^{E_{x}} / K_{g} \text { if } E_{1} \geq 0 \\
0 \text { else }
\end{array}\right. \\
i_{g c}=\left\{\begin{array}{l}
0 \text { if } v_{g c}<V_{a} \\
\frac{v_{g c} V_{a}}{R_{g k}} \text { else }
\end{array}\right.
\end{gathered}
$$

with

$$
E_{1}=\frac{v_{p c}}{K_{p}} \ln \left(1+\exp \left(K_{p}\left(\frac{1}{\mu}+\frac{v_{g c}+V_{c t}}{\sqrt{K_{v b}+v_{p c}^{2}}}\right)\right)\right.
$$

The parameters set $\theta=\left(\mu, E_{x}, K_{g}, K_{p}, K_{v b}, V_{c t}, V_{a}, R_{g k}\right)$ is retrieved from the datasheet [13] through a least squares minimization. This allows the modelling of the triode as a dissipative component in the PHS formalism, with $w=$ $\left(\begin{array}{c}v_{p c} \\ v_{g c}\end{array}\right)$ and $z_{\theta}(w)=\left(\begin{array}{c}i_{p c} \\ i_{g c}\end{array}\right)$.

\subsubsection{Transformer and feedback loop}

The oscillation starts with a voltage noise $V_{\text {start }}$ at the triode grid, which is amplified and filtered by the LC circuit at the triode plate before being re-injected in the grid through a transformer of ratio $M$. Under open circuit (negligible influence of the following stages) and small-signal (possible linearization around an operating point) hypotheses, the oscillator can be represented as a system of input $V_{\text {start }}$ and

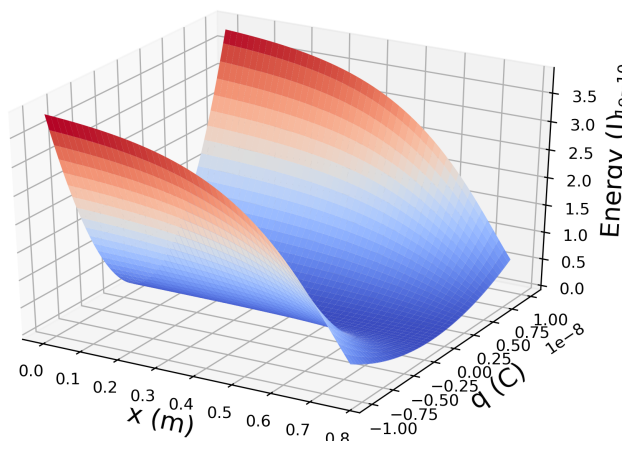

Fig. 6. Energy function of the variable capacitor.

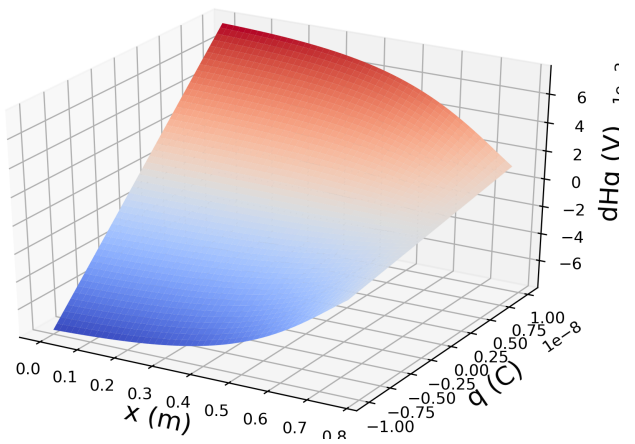

Fig. 7. Constitutive law of the variable capacitor. 
output $V_{\text {out }}$ (fig. 8, with $\mu$ the triode amplification factor and $r a$ the anode resistor). The plate load is constituted by a LC parallel circuit, with impedance ( $s$ is the Laplace variable)

$$
Z=\frac{s L}{1+s^{2} L C}
$$

Moreover, as $V_{\text {bias }}$ is constant, $\Delta V_{\text {out }}=-[Z /(Z+r a)] \Delta v_{p c}$ where operator $\Delta$ denotes variations around the operating point. Considering the triode amplification factor definition, this yields $\Delta V_{\text {out }}=[Z /(Z+r a)] \mu\left(M \Delta V_{\text {out }}+V_{\text {start }}\right)$, so that

$$
\frac{\Delta V_{\text {out }}}{V_{\text {start }}}=\frac{Z \mu}{Z(1-\mu M)+r a} .
$$

The poles of transfer function (19) are found to be the roots (in $s$ ) of the characteristic equation

$$
s^{2}+s \frac{1-\mu M}{r a C}+\omega_{0}^{2}=0
$$

with $\omega_{0}^{2}=\frac{1}{L C}$. The condition for the system to start oscillating is that a complex pole has a positive real part. This leads to

$$
\frac{1}{\mu} \leq M<\frac{1}{\mu}+\frac{2 \omega_{0} C}{g m}
$$

where $g m=\mu / r a$ is the triode transconductance. The closer to $1 / \mu$ is chosen $M$, the more stable is the oscillation amplitude.

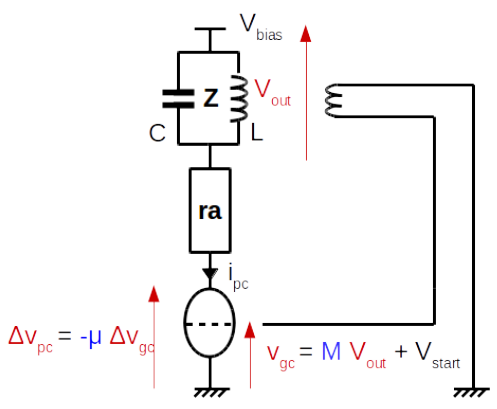

Fig. 8. Schematic of the simplified oscillator.

\subsubsection{Interconnection}

Replacing parallel capacitors $C_{1} / / C_{2}$ with equivalent capacitor $C_{3}$ and $C_{13} / / C_{14} / / C_{15}$ with equivalent capacitor $C_{8}$, the oscillator is realizable and can be represented as the following PHS:

$$
\left(\begin{array}{c}
v_{L} \\
i_{C 8} \\
i_{C 3} \\
v_{R 1} \\
v_{p c} \\
v_{g c} \\
-I_{\text {bias }} \\
-I_{\text {start }}
\end{array}\right)=\left(\begin{array}{cccccccc}
0 & 1 & 0 & 0 & 0 & 0 & 0 & 0 \\
-1 & 0 & 0 & 0 & 1 & -M & 0 & 0 \\
0 & 0 & 0 & -1 & 1 & 1 & 0 & 0 \\
0 & 0 & 1 & 0 & 0 & 0 & 0 & 0 \\
0 & -1 & -1 & 0 & 0 & 0 & 1 & 0 \\
0 & M & -1 & 0 & 0 & 0 & 0 & 1 \\
0 & 0 & 0 & 0 & -1 & 0 & 0 & 0 \\
0 & 0 & 0 & 0 & 0 & -1 & 0 & 0
\end{array}\right) \cdot\left(\begin{array}{c}
i_{L} \\
v_{C 8} \\
v_{C 3} \\
i_{R 1} \\
i_{p c} \\
i_{g c} \\
V_{\text {bias }} \\
V_{\text {start }}
\end{array}\right)
$$

\subsection{Numerical experiments}

We now simulate the complete variable oscillator, sliding the ribbon from $A 1$ to $A 6$. The simulation is performed ac- cording to the power-balanced numerical scheme presented in [2] (see also the PyPHS library [10]). In order to observe the frequency changes, we choose a carrier frequency $F=10 \mathrm{kHz}$ (instead of the actual $80 \mathrm{kHz}$ ) and perform the sweep in $2 \mathrm{~ms}\left(\mathrm{vel}_{1}\right)$ or in $0.5 \mathrm{~s}\left(\mathrm{vel}_{2}\right)$. The simulation parameters are presented in table 1. Due to important nonlinearities of some components, a large sample-rate is chosen

\begin{tabular}{|c|c|c|c|c|c|}
\hline $6 \mathrm{F5}$ & $\mu$ & $E_{x}$ & $K_{g}$ & $K_{p}$ & $K_{v b}$ \\
\hline & 98 & 1.6 & 2614 & 905 & 1.87 \\
\hline & $V_{c t}$ & $V_{a}$ & $R_{g k}$ & & \\
\hline & 0.5 & 0.33 & 1300 & & \\
\hline$R_{1}$ & $L$ & $C_{1} / / C_{2}$ & $C_{13} / / C_{14}$ & $\boldsymbol{V}_{\text {bias }}$ & $M$ \\
\hline $7.5 \mathrm{k} \Omega$ & $2 \mathrm{mH}$ & $0.22 \mu F$ & $440 p F$ & $90 \mathrm{~V}$ & $\frac{1}{\mu}$ \\
\hline
\end{tabular}
to avoid any aliasing. Table 2 recaps the components values. We denote $C_{1} / / C_{2}=C_{3}$ and $C_{13} / / C_{14} / / C_{15}=C_{8}$. Figs. 9 and

\begin{tabular}{cccc}
\hline $\boldsymbol{F} \boldsymbol{s}$ & $\boldsymbol{F}$ & vel $_{\mathbf{1}}$ & vel $_{\mathbf{2}}$ \\
\hline $768 \mathrm{kHz}$ & $10 \mathrm{kHz}$ & $385 \mathrm{~m} / \mathrm{s}$ & $1.54 \mathrm{~m} / \mathrm{s}$
\end{tabular}

Table 1. Simulation parameters.

Table 2. Components parameters values.

10 show the observed flows and efforts of the oscillator as the ribbon slides. Figs. 11 and 12 show the states of the different storage components, reflecting the frequency changes. Figs. 14 and 15 show the power balance of the complete system during the simulation. Fig. 13 shows the spectrogram of the output voltage, suggesting that the harmonic distortion is sufficiently negligible. It is also worth noting that the PHS formalism gives access to other physical parameters of the ribbon, which would otherwise prove difficult to measure. Indeed, the quantity

$$
\nabla_{x} H_{c a p}(x, q)=\frac{-q^{2} C^{\prime}(x)}{2 C^{2}(x)}
$$

is the force produced by the ribbon displacement (see $\mathrm{Re}$ mark 3) ; figs. 16 and 17 show that the values taken by this force during the sweep are negligible no matter the sweep velocity.

\begin{tabular}{cccc}
\hline & $q C_{3}$ ref & $q C_{8}$ ref & $\phi r e f$ \\
\hline vel $_{1}$ & $4.60 .10^{-6} \mathrm{C}$ & $3.13 .10^{-8} \mathrm{C}$ & $4.60 .10^{-6} \mathrm{~Wb}$ \\
\hline vel $_{2}$ & $4.58 .10^{-6} \mathrm{C}$ & $4.76 .10^{-8} \mathrm{C}$ & $4.58 .10^{-6} \mathrm{~Wb}$
\end{tabular}

Table 3. Reference values.

\section{Interpretation and discussion}

Due to the nature of the Ondes Martenot instrument (rare, fragile and expensive), setting up extensive measurements to evaluate the accuracy of the oscillator model is a complex operation which is still ongoing. However, a preliminary observation is that with the chosen parameters, the oscillation is quasi-sinusoidal (less that $0.1 \%$ harmonic distortion for 
the second harmonic), which corresponds to observations made in [14] : "on the whole ribbon range, the sinusoidal quality of the signal produced by the oscillator is excellent". Moreover, the behaviours of the components are not affected by the ribbon displacement speed and there is no latency between the ribbon displacement and the frequency changes. This suggests that for future simulations of the complete circuit, the oscillator could be modelled with a frequency-controlled sinusoidal voltage generator, which would save computation time. It can be noted though that the output voltage of the oscillator slightly decreases as

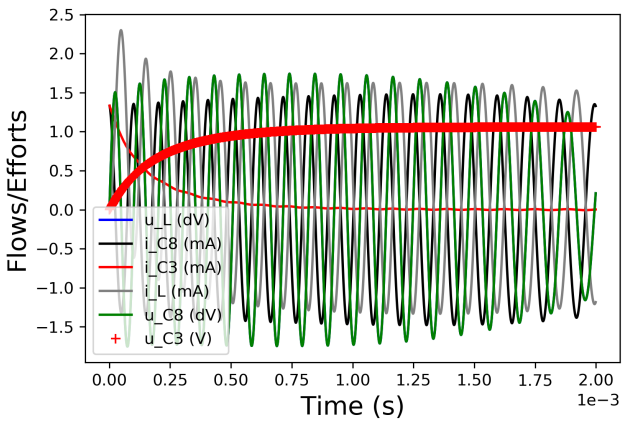

Fig. 9. Velocity $v e l_{1}=384 \mathrm{~m} / \mathrm{s}$ : simulated flows and efforts of the storage components, for a carrier frequency $F=10 \mathrm{kHz}$, during a sweep from $A 1$ to $A 6$.

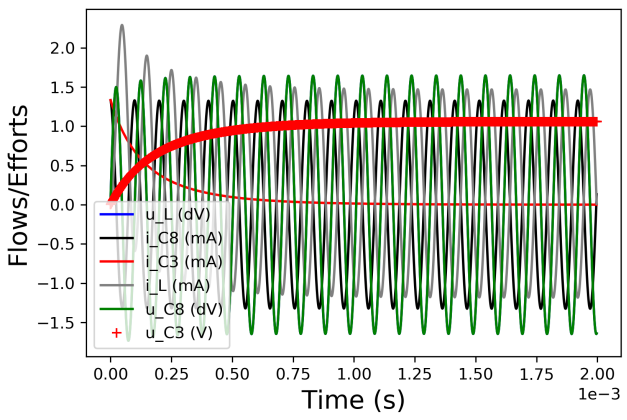

Fig. 10 . Velocity $v e l_{2}=1.54 \mathrm{~m} / \mathrm{s}$ : simulated flows and efforts of the storage components, for a carrier frequency $F=10 \mathrm{kHz}$, during a sweep from $A 1$ to $A 6$ zoomed on the first $2 \mathrm{~ms}$.

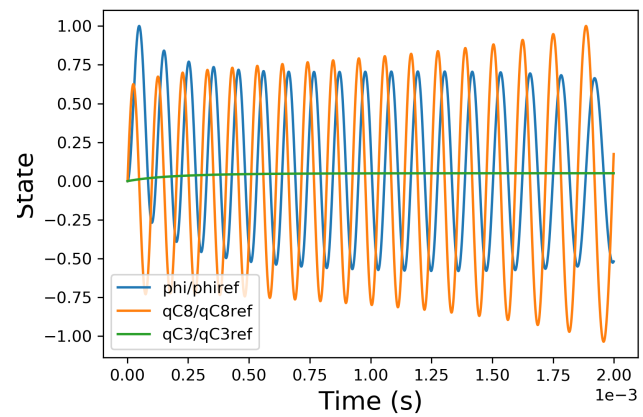

Fig. 11 . Velocity $v e l_{1}=384 \mathrm{~m} / \mathrm{s}$ : simulated states of the storage components during a sweep from $A 1$ to $A 6$. Table 3 shows the reference values.

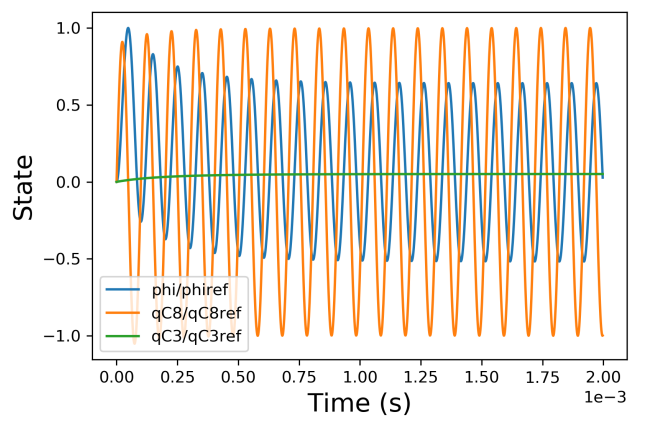

Fig. 12 . Velocity $v^{2} l_{2}=1.54 \mathrm{~m} / \mathrm{s}$ : simulated states of the storage components during a sweep from $A 1$ to $A 6$ zoomed on the first $2 \mathrm{~ms}$. Table 3 shows the reference values.

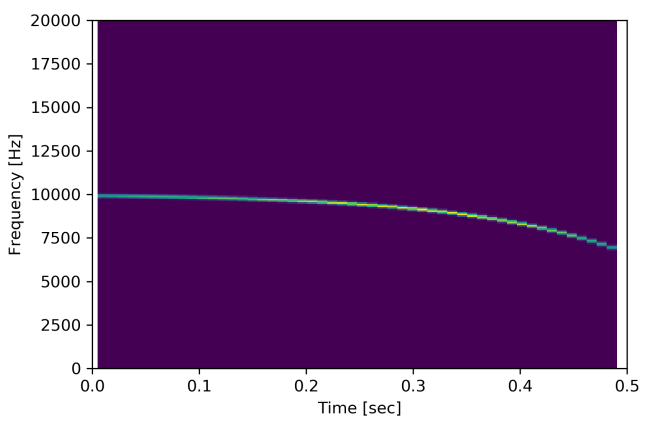

Fig. 13. Spectrogram of the output voltage during a sweep from A1 to $A 6$.

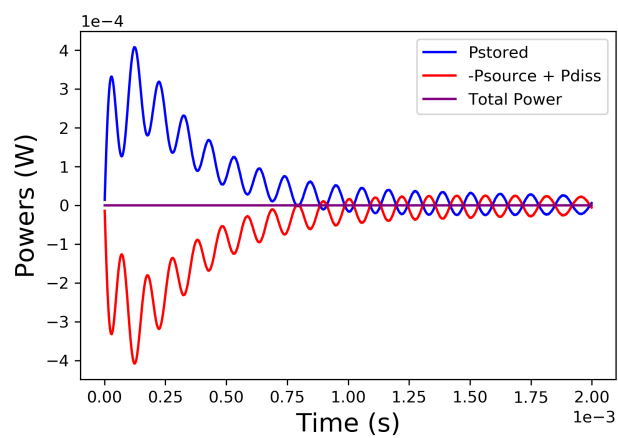

Fig. 14. Velocity $v e l_{1}=384 \mathrm{~m} / \mathrm{s}$ : power balance of the system during a sweep from $A 1$ to $A 6$.

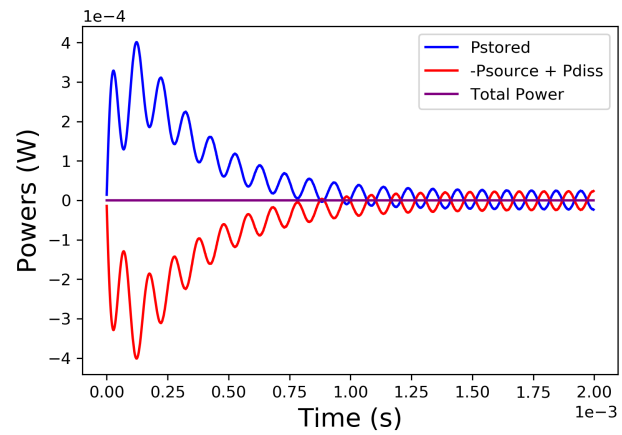

Fig. 15 . Velocity $v^{2} l_{2}=1.54 \mathrm{~m} / \mathrm{s}$ : power balance of the system during a sweep from $A 1$ to $A 6$ zoomed on the first $2 \mathrm{~ms}$. 
the heard frequency increases, which is not the case in real Ondes Martenot. Real instruments have a higher frequency carrier, therefore frequency modulations are relatively less important. The oscillator capacitance varies less and consequently the triode amplification, which depends on its load, is stable during the whole sweep. A second observation is that consistently with the PHS formalism, the power balance is preserved despite the nonlinearities of some components. A third observation is that the mechanical force produced by the ribbon displacement is extremely low (less than $10 \mathrm{nN}$ ), thus presumably not detectable by the musician. This is likely to be what Maurice Martenot intended as the interaction between the player and the instrument should be as smooth as possible.

\section{Conclusion and perspectives}

In this paper, a refined simulation of the ribbon-controlled oscillator of the Ondes Martenot has been proposed, allowing a numerical investigation of this circuit. It is based on an energy-balanced modelling adapted to LTI (capacitors, resistor, inductor) and non LTI (vacuum tube and the multiphysical time-varying capacitor mechanically-driven by a ribbon) components. One contribution of this paper is the design of a method to compute equivalent energy functions of groups of components when required, to derive a PHS statespace realization of a global circuit. This method reveals that LTI and variable capacitors in parallel build an equiva-

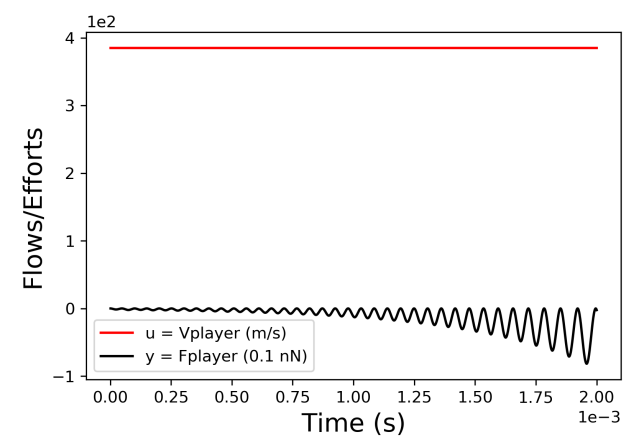

Fig. 16. Velocity $v e l_{1}=384 \mathrm{~m} / \mathrm{s}$ : mechanical flow and effort during a sweep from $A 1$ to $A 6$.

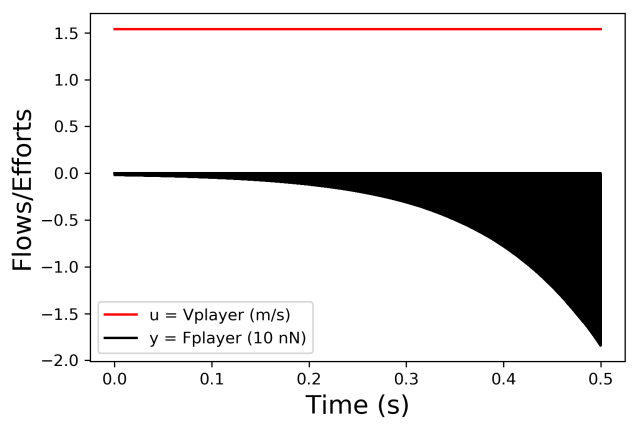

Fig. 17. Velocity $v e l_{2}=1.54 \mathrm{~m} / \mathrm{s}$ : mechanical flow and effort during a sweep from $A 1$ to $A 6$. lent bi-variate capacitor, depending on an electric state (total charge) and a space state (ribbon position). As a second contribution, the ideal energy function for the ribbon-controlled equivalent capacitor is derived in correspondence with the target keyboard designed by Martenot. Numerical experiments on the nonlinear time-varying circuit lead to expected observations: (1) the combination of the triode amplification and the LC-resonator produces a quasi-sinusoidal oscillation with a stable amplitude for a static configuration; (2) the mechanical force produced by the variable capacitor due to the ribbon displacement is undetectable by the musician (less than $10 \mathrm{nN}$ ) for over-speed movement ( $300 \mathrm{~m} / \mathrm{s}$ ); (3) the latency between the instantaneous frequency and the ribbon position is also undetectable. This corroborates that the Martenot's ribbon-controlled circuit is close to an ideal oscillator.

Further work aims to model and simulate the complete Martenot instrument, including all its stages and the diffuseurs. Furthermore, for real-time sound synthesis purposes, the examination of anti-aliasing methods [15] could be profitable to handle the nonlinear heterodyne process at reasonable sampling rates.

\section{Acknowledgments}

This work was funded by the Collegium Musicae and is supported by the project ANR-16-CE92-0028, entitled Interconnected Infinite-Dimensional systems for Heterogeneous Media, INFIDHEM, financed by the French National Research Agency (ANR).

\section{References}

[1] Leipp, E., "Les Ondes Martenot," bulletin du GAM no60, 1972.

[2] Falaize, A., Modélisation, simulation, génération de code et correction de systèmes multi-physiques audios: Approche par réseau de composants et formulation Hamiltonienne à Ports, Ph.D. thesis, Université Pierre \& Marie Curie-Paris 6, 2016.

[3] Duindam, V., Macchelli, A., Stramigioli, S., and Bruyninckx, H., Modeling and control of complex physical systems: the port-Hamiltonian approach, Springer Science \& Business Media, 2009.

[4] van der Schaft, A., Jeltsema, D., et al., "PortHamiltonian systems theory: An introductory overview," Foundations and Trends® in Systems and Control, 1(2-3), pp. 173-378, 2014.

[5] Hélie, T., Falaize, A., and Lopes, N., "Systèmes Hamiltoniens à Ports avec approche par composants pour la simulation à passivité garantie de problèmes conservatifs et dissipatifs," in Colloque National en Calcul des Structures, volume 12, 2015.

[6] Falaize, A. and Hélie, T., "Passive guaranteed simulation of analog audio circuits: A port-Hamiltonian approach," Applied Sciences, 6(10), p. 273, 2016.

[7] Brockett, R. W., Finite dimensional linear systems, volume 74, SIAM, 2015. 
[8] Van der Schaft, A., "A realization procedure for systems of nonlinear higher-order differential equations," IFAC Proceedings Volumes, 20(5), pp. 85-90, 1987.

[9] Sarti, A. and De Poli, G., "Toward nonlinear wave digital filters," IEEE Transactions on Signal Processing, 47(6), pp. 1654-1668, 1999.

[10] Falaize, A. and Hélie, T., "PyPHS: Passive modeling and simulation in python," https://pyphs. github.io/pyphs/, 2016.

[11] Courrier, T., "Analyse de fonctionnement Onde 169," 2012, unpublished document, Musée de la musique.

[12] Cohen, I., Modélisation, analyse et identification de circuits non linéaires: Application aux amplificateurs guitare à lampes pour la simulation en temps réel, Ph.D. thesis, Université Pierre \& Marie Curie-Paris 6, 2012.

[13] Tung-Sol, "6F5 typical operating conditions and characteristics," https://frank.pocnet.net/ sheets/127/6/6F5.pdf, 1942.

[14] Courrier, T., “Analyse de fonctionnement Onde 15,' 2012, unpublished document, Musée de la musique.

[15] Muller, R. and Hélie, T., "Trajectory anti-aliasing on guaranteed-passive simulation of nonlinear physical systems," in 20th International Conference on Digital Audio Effects (DAFx-17), 2017.

[16] Itoh, T. and Abe, K., "Hamiltonian-conserving discrete canonical equations based on variational difference quotients," Journal of Computational Physics, 76(1), pp. 85-102, 1988.

\section{Appendix}

\section{A.1 PHS formalism : example}

Consider a linear parallel RLC circuit (fig.18). The ca-

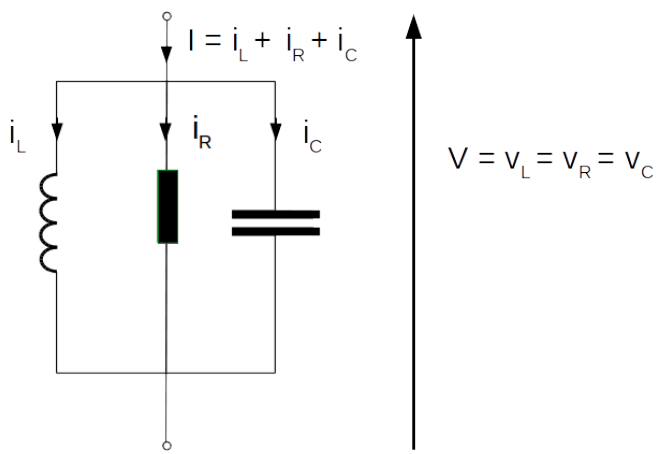

Fig. 18. Parallel RLC

pacitor $C$ and the inductor $L$ are storage components whose states are given by the variables $q$ (charge) and $\phi$ (magnetic flux) respectively ; the resistor $R$ is a dissipative component described by Ohm's law. The system is current (flow) controlled, its associated output is a voltage (effort). Table 4 recaps the variables and the constitutive laws of the three

\begin{tabular}{ccccc}
\hline & $x$ & $\frac{d x}{d t}$ & $H(x)$ & $\nabla H(x)$ \\
\hline $\mathbf{C}$ & $q$ & $\dot{q}=i_{C}$ & $\frac{q^{2}}{2 C}$ & $\frac{q}{C}=v_{C}$ \\
$\mathbf{L}$ & $\phi$ & $\dot{\phi}=v_{L}$ & $\frac{\phi^{2}}{2 L}$ & $\frac{\phi}{L}=i_{L}$ \\
\hline & $w$ & & $z(w)$ \\
\hline $\mathbf{R}$ & & $v_{R}$ & & $\frac{v_{R}}{R}=i_{R}$
\end{tabular}

Table 4. State variables and constitutive laws for a linear parallel RLC circuit.

components. Kirchoff's laws in receptor convention yield

$$
\left(\begin{array}{c}
i_{C} \\
v_{L} \\
v_{R} \\
-V
\end{array}\right)=\left(\begin{array}{cccc}
0 & -1 & -1 & 1 \\
1 & 0 & 0 & 0 \\
1 & 0 & 0 & 0 \\
-1 & 0 & 0 & 0
\end{array}\right) \cdot\left(\begin{array}{c}
v_{C} \\
i_{L} \\
i_{R} \\
I
\end{array}\right)
$$

Currents are flows and voltages are efforts, therefore their products are powers. Eq. (5) is thus naturally retrieved. Note that in this simple example, the matrix $S$ is sparse with constant coefficients, but the properties of the PHS formalism hold for nonlinear or coupled systems which yield more complex matrices.

\section{A.2 PHS : numerical scheme for simulations}

The PHS formalism guarantees the passivity of the system in continuous time. Introducing discrete gradient in the numerical scheme [16] allows to preserve this passivity property in discrete time, therefore granting the stability of the simulation as well. Here a one-step numerical scheme is used, yielding

$$
x(k+1)=x(k)+\delta x(k)
$$

In the mono-variate storage component case $(H(x)=$ $\sum_{n=1}^{N} H_{n}\left(x_{n}\right)$ where $N$ is the storage components number), the discrete gradient $[\bar{\nabla} H(x, \delta x)]_{n}$ is defined by

$$
[\bar{\nabla} H(x, \delta x)]_{n}= \begin{cases}\frac{H_{n}\left(x_{n}+\delta x_{n}\right)-H_{n}\left(x_{n}\right)}{\delta x_{n}} & \text { if } \delta x_{n} \neq 0, \\ H_{n}^{\prime}\left(x_{n}\right) & \text { else. }\end{cases}
$$

The discrete energy variation is retrieved by chain derivation:

$$
\frac{\delta E(k)}{t_{s}}=\bar{\nabla} H(x, \delta x(k))^{\top} \frac{\delta x(k)}{t_{s}}
$$

where $t_{s}$ is the sampling period. The simulation is achieved replacing $\frac{d x}{d t}$ with $\frac{\delta x(k)}{t_{s}}$ and $\nabla H(x)$ with $\bar{\nabla} H(x, \delta x)$ in (3). This yields a dynamic equation of the form

$$
\delta x(k)=t_{s} f_{k}(x(k), \delta x(k))
$$

where $f_{k}$ is a function depending on $H, z, u$ and $S$. Its solving (using Newton-Raphson method for instance) allows to compute $x(k+1)$ and $y(k)$.

\section{A.3 Equivalent component method : examples}

The method steps (sec. 3) are detailed on two examples:

(LTI) linear time-invariant capacitors with capacitances $C_{k}$, (in order to illustrate how the standard results of section 2 are restored, and to be compared at each step to) 

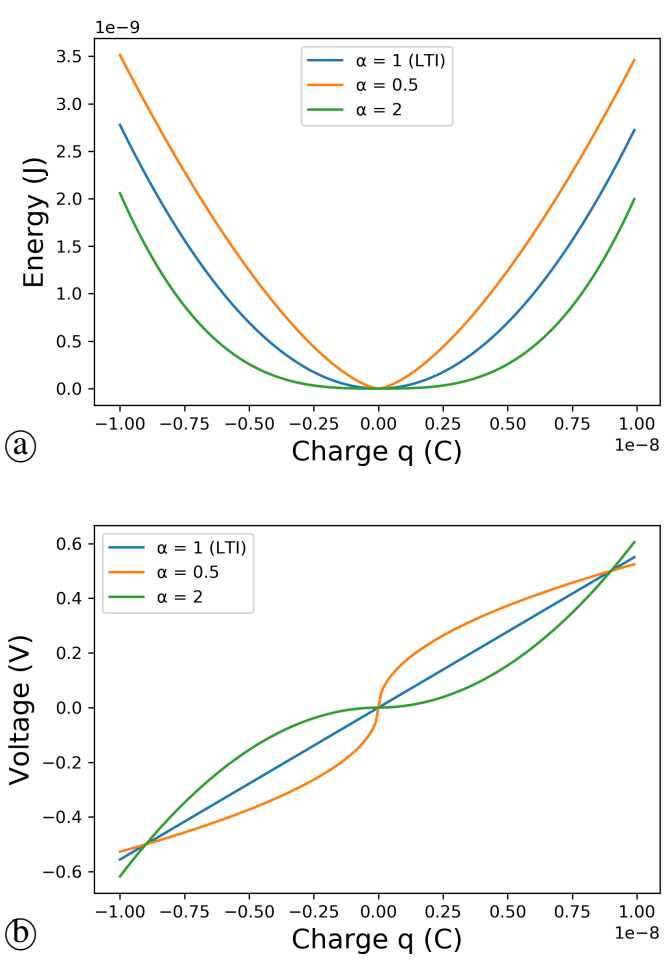

Fig. 19. (NL) Energy functions $H_{k}$ (top) and voltage functions $H_{k}^{\prime}$ (bottom) for various degrees $\alpha\left(q_{0}=0.9 \cdot 10^{-8} \mathrm{C}\right.$ and $\left.V_{k}=0.5 \mathrm{~V}\right)$.

(NL) capacitors with homogeneous laws of common degree $\alpha>0$ (nonlinear for $\alpha \neq 1$, see Fig.19).

\begin{tabular}{|l||l|l|}
\hline Label & Energy function $H_{k}$ & Voltage function $H_{k}^{\prime}$ \\
\hline \hline LTI & $H_{k}\left(q_{k}\right)=\frac{q_{k}^{2}}{2 C_{k}}$ & $v_{k}=H_{k}^{\prime}\left(q_{k}\right)=\frac{q_{k}}{C_{k}}$ \\
\hline NL & $H_{k}\left(q_{k}\right)=E_{k}\left|\frac{q_{k}}{q_{0}}\right|^{1+\alpha}$ & $v_{k}=H_{k}^{\prime}\left(q_{k}\right)=V_{k}\left[\frac{q_{k}}{q_{0}}\right]^{\alpha}$ \\
\hline
\end{tabular}

In the model (NL), $[x]^{\alpha}=\operatorname{sign}(x)|x|^{\alpha}$ denotes the signed power function and constant values $q_{0}$ (charge), $E_{k}$ (energy) and $V_{k}$ (voltage) are related by

$$
V_{k}=(1+\alpha) E_{k} / q_{0}
$$

This leads to the following sequence of derivations.

Step 1. Eq. (7) yields $q_{k}=H_{k}^{\prime-1}\left(v_{k}\right)$ with

$$
\begin{aligned}
& \text { (LTI): } H_{k}^{\prime-1}\left(v_{k}\right)=C_{k} v_{k}, \\
& \text { (NL): } H_{k}^{\prime-1}\left(v_{k}\right)=q_{0}\left[\frac{v_{k}}{V_{k}}\right]^{1 / \alpha},
\end{aligned}
$$

and Eq. (8) yields $q_{C}=Q_{C}\left(v_{C}\right)$ with $Q_{C}\left(v_{C}\right):=\left[H_{A}^{\prime-1}+\right.$ $\left.H_{B}^{\prime-1}\right]\left(v_{C}\right)$,

$$
\text { (LTI): } \begin{aligned}
Q_{C}\left(v_{C}\right) & =\left(C_{A}+C_{B}\right) v_{C} \\
& =q_{C}\left(\frac{v_{C}}{V_{C}}\right]^{1 / \alpha},
\end{aligned}
$$$$
\text { with } V_{C}=\left[V_{A}^{-1 / \alpha}+V_{B}^{-1 / \alpha}\right]^{-\alpha} \text {. }
$$

Step 2. Eq. (9) yields $v_{C}=Q_{C}^{-1}\left(q_{C}\right)$ with

$$
\begin{aligned}
& \text { (LTI): } Q_{C}^{-1}\left(q_{C}\right)=\frac{q_{C}}{C_{A}+C_{B}} \\
& \text { (NL): } Q_{C}^{-1}\left(q_{C}\right)=V_{C}\left[\frac{q_{C}}{q_{0}}\right]^{\alpha} .
\end{aligned}
$$

Step 3. Eq. (10) yields

$$
\begin{aligned}
& \text { (LTI): } H_{C}\left(q_{C}\right)=\frac{\left(\frac{C_{A} q_{C}}{C_{A}+C_{B}}\right)^{2}}{2 C_{A}}+\frac{\left(\frac{C_{B} q_{C}}{C_{A}+C_{B}}\right)^{2}}{2 C_{B}} \\
& =\frac{q_{C}^{2}}{2\left(C_{A}+C_{B}\right)} \\
& (\mathrm{NL}): H_{C}\left(q_{C}\right)=\sum_{k=A, B} E_{k}\left|\frac{q_{0}\left[\frac{V_{C}\left[\frac{q_{C}}{q_{0}}\right]^{\alpha}}{V_{k}}\right]^{1 / \alpha}}{q_{0}}\right|^{1+\alpha} \\
& =E_{C}\left|\frac{q_{c}}{q_{0}}\right|^{1+\alpha}
\end{aligned}
$$

where $E_{C}$ is found to be related to $V_{C}$ as $E_{k}$ to $V_{k}$ in Eq. (27)

Note that the equivalent laws for (LTI) and (NL) have the same expression. This is not the case in general. 